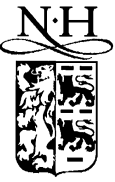

ELSEVIER

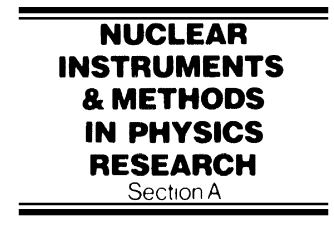

www.elsevier.nl/locate/nima

\title{
Performance of large area CsI-RICH prototypes for ALICE at LHC
}

\author{
for the ALICE collaboration
}

\author{
A. Di Mauro ${ }^{\mathrm{a}, *}$, D. Cozza $^{\mathrm{b}}$, M. Davenport ${ }^{\mathrm{a}}$, D. Di Bari ${ }^{\mathrm{b}}$, D. Elia ${ }^{\mathrm{b}}$, P. Martinengo $^{\mathrm{a}}$,

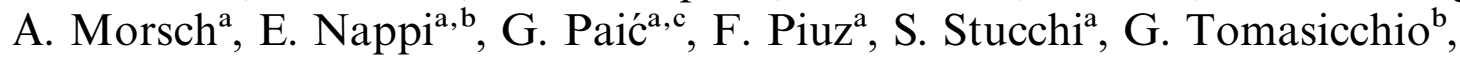 \\ T.D. Williams ${ }^{\mathrm{a}}$ \\ ${ }^{\text {a } C E R N, ~ E u r o p e a n ~ O r g a n i z a t i o n ~ f o r ~ N u c l e a r ~ R e s e a r c h, ~} 1211$ Geneva, Switzerland \\ ${ }^{\mathrm{b}}$ INFN and Dipartimento Interateneo di Fisica dell'Universitá di Bari, Bari, Italy \\ 'Ohio State University, Columbus, OH, USA
}

\begin{abstract}
We present the performances of large area CsI-RICH prototypes obtained in single-particle events. The differential quantum efficiency of the photocathodes has been deduced from Cherenkov rings by means of two different procedures: a direct measurement with a thin $\mathrm{NaF}$ radiator and a Monte Carlo-based estimation for a $\mathrm{C}_{6} \mathrm{~F}_{14}$ radiator. A factor of merit of $45 \mathrm{~cm}^{-1}$ has been found for the typical detector configuration. Two angle reconstruction algorithms have been used and the different errors affecting the Cherenkov angle resolution have been estimated combining the analytical treatment and the Monte Carlo simulation. Also the dependence on radiator thickness, Cherenkov ring radius, chamber voltage and particle incidence angle has been studied. (C) 1999 Elsevier Science B.V. All rights reserved.
\end{abstract}

\section{Introduction}

In the framework of the development of a large area CsI-RICH detector for ALICE (A Large Ion Collider Experiment [1]) at LHC, various prototypes, having active areas up to $\sim 1 \mathrm{~m}^{2}$, have been studied in beam tests aiming at the evaluation of two fundamental properties: the CsI photocathode (PC) quantum efficiency (QE) and the Cherenkov angle resolution [2].

The differential QE of CsI PC's is usually measured in laboratory with dedicated UV-setups;

\footnotetext{
* Corresponding author.
}

however, these can be operated generally only with small size samples. In the present work we demonstrate how the QE dependence on wavelength of a large-area CsI PC can be evaluated from the measurement of the Cherenkov light in a RICH detector.

In the last part of the paper, to characterize the performance in terms of particle identification capability, the main results of a detailed examination of the angular resolution will be discussed.

\section{Experimental setup}

The detector configuration is schematically shown in Fig. 1, while a complete description of the 


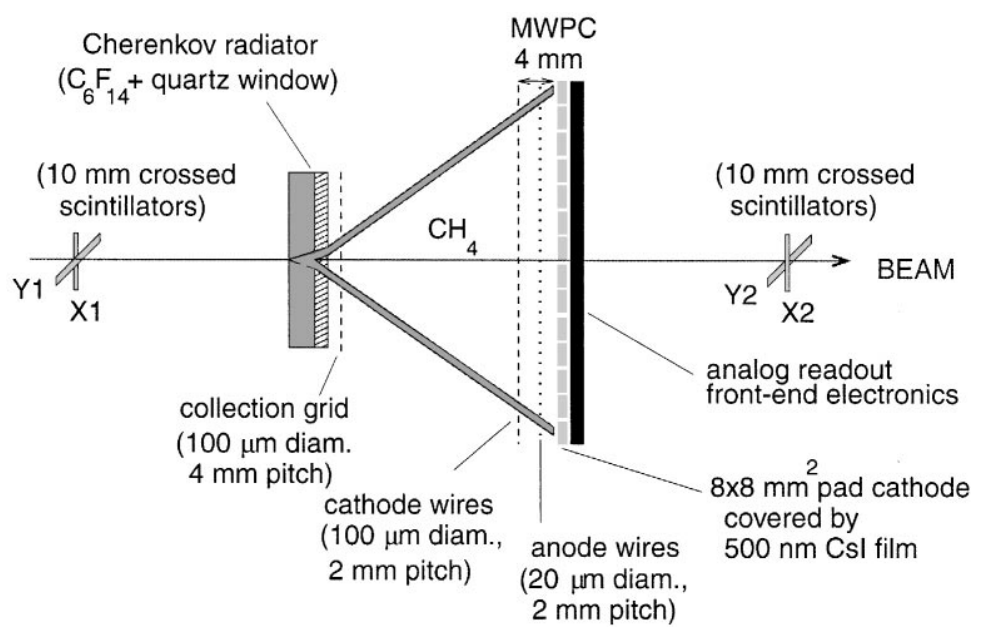

Fig. 1. Scheme of the CsI-RICH prototype geometry.

test-beam setup can be found in Ref. [2]. The RICH detector has a proximity focusing geometry with a multiwire chamber having a CsI photocathode segmented into $8 \times 8 \mathrm{~mm}^{2}$ pads, with a 2 -d analog multiplexed readout.

Tests of a prototype of $32 \times 30 \mathrm{~cm}^{2}$ active area (proto-1) have been performed in the CERN PS/T11 test-beam composed of protons and pions in the $1-3.5 \mathrm{GeV} / c$ momentum range. Two crossed plastic scintillator doublets, $2 \mathrm{~m}$ apart, define the trigger geometry covering an area of $1 \mathrm{~cm}^{2}$ of the detector, with a beam divergence $\leq 1 \mathrm{mrad}$. A time-of-flight system based on two scintillators, $10 \mathrm{~m}$ apart, provides offline discrimination between protons and pions. Proto- 1 can be equipped with a $\mathrm{NaF}$ radiator or an adjustable thickness $\mathrm{C}_{6} \mathrm{~F}_{14}$ liquid radiator with a $3 \mathrm{~mm}$ quartz window.

A larger prototype composed of 4 PC's of $64 \times$ $40 \mathrm{~cm}^{2}$ (proto-2), for a total size of $\sim 1.3 \times 0.8 \mathrm{~m}^{2}$, has been tested at the CERN SPS/H4 $350 \mathrm{GeV} / c$ pions test-beam. In this case the two crossed scintillator doublets defining the beam are about $10 \mathrm{~m}$ apart, while the radiator tray has a fixed thickness of $10 \mathrm{~mm}$ with a $5 \mathrm{~mm}$ quartz window.

\section{Event analysis}

Depending on detector configuration, beam momentum and particle hit point, a Cherenkov fiducial zone is located in the pad plane: it is an annular region where all the Cherenkov photons may be detected. For QE evaluation purposes it is important to establish such a fiducial zone, since the radiation spectrum is well defined inside it.

A fundamental step of the event processing is the cluster deconvolution: it is expected that a certain fraction of single photoelectron clusters may overlap originating larger clusters with multiple local pulse-height $(\mathrm{PH})$ maxima. A contribution from secondary processes is also expected; however due to the small anode-cathode gap $(2 \mathrm{~mm})$ and the pad size $(8 \mathrm{~mm})$, feedback photons are mostly contributing to the same cluster created by the primary avalanche.

Clusters are first counted as raw clusters and after image processing as resolved clusters. Raw clusters are classified into three categories depending on the size and the PH distribution (Fig. 2):

- Class 1: clusters of one, two or three pads (row or L-shaped) when the PH maximum is in the central pad, assumed to correspond to single photoelectrons;

- Class 2: clusters of three pads when the PH maximum is not in the central pad, assumed to correspond to two photoelectrons;

- Class 3: clusters of more than three pads, assumed to correspond to two or more photoelectrons. 

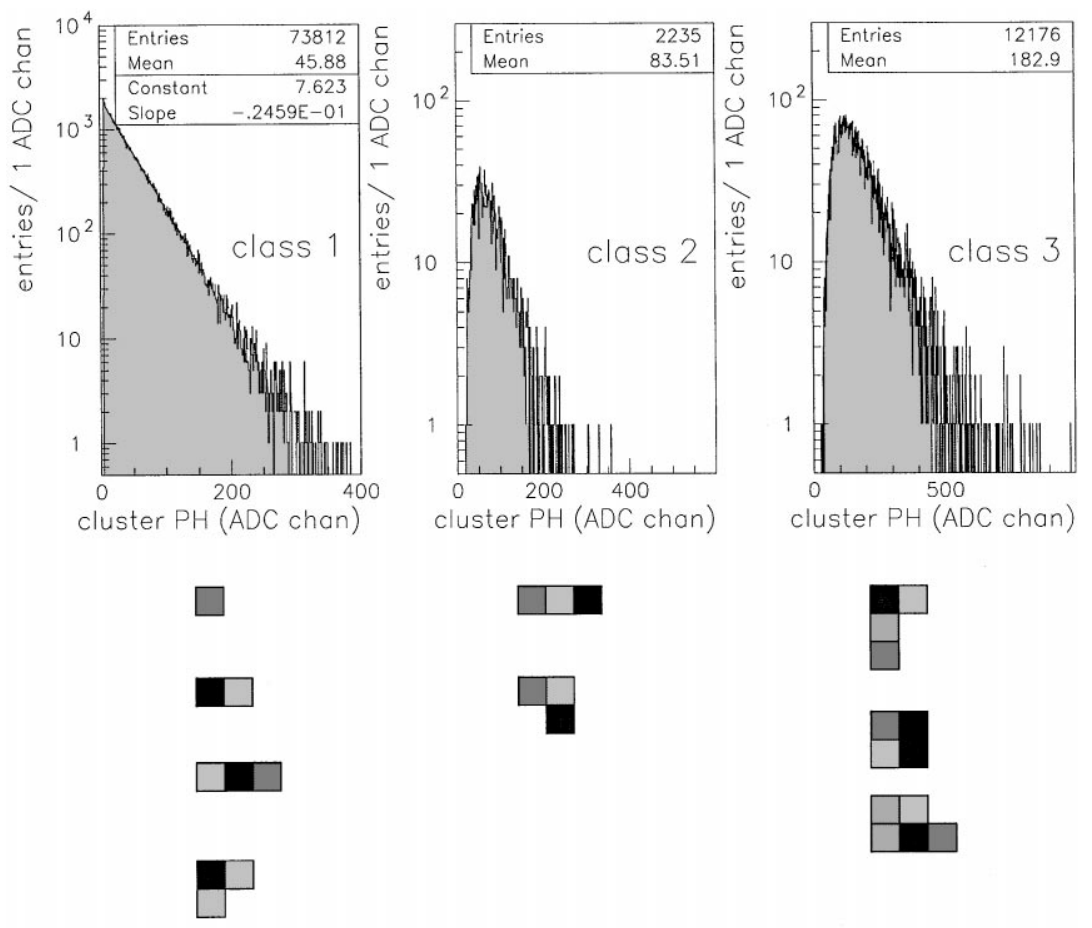

class 1

class 2

class 3

Fig. 2. (top) The total PH distributions of the three classes of raw clusters and (bottom) the geometrical features for each class (the darker the grey tone, the larger the pad $\mathrm{PH}$ ).

Fig. 2 also presents the cluster PH distribution for each class, from test-beam events with proto-2 operated with $\mathrm{CH}_{4}$ at $2100 \mathrm{~V}$. In these conditions the class $1 \mathrm{PH}$ distribution is generally exponential and the single-electron mean PH $A_{0}$, namely the chamber gain, can be deduced from the slope of the fit (also reported in the figure). The average and the shape of the $\mathrm{PH}$ distributions relative to class 2 and 3 clusters, compared to that of class 1 , indicate clearly the contribution of two or more photoelectrons.

In the majority of the cases class 2 and 3 clusters can be resolved into smaller sized clusters by an algorithm producing $n$ resolved clusters when $n$ local $\mathrm{PH}$ maxima are found in the original raw cluster.

Finally, the impact position of particles and photons, for clusters of more than one pad, is evaluated with the centroid finding method, while for single-pad clusters it is assumed to be in the pad centre.

\section{CsI QE evaluation in Cherenkov events}

The knowledge of the spectrum of the Cherenkov radiation and the transmission of the media traversed by the photons has been exploited to evaluate the differential QE of large area CsI PCs with two different approaches. The first allows a direct measurement of the CsI QE curve making use of the large dispersion of the refractive index of a $\mathrm{NaF}$ radiator [3]. The other is based on the simulation of Cherenkov events aiming at the tuning of the quantum response through the reproduction of main quantities evaluated in beam tests.

\subsection{Direct measurement with a NaF radiator}

The number of photons having an energy within $E, E+\mathrm{d} E$, reaching the photocathode, can 


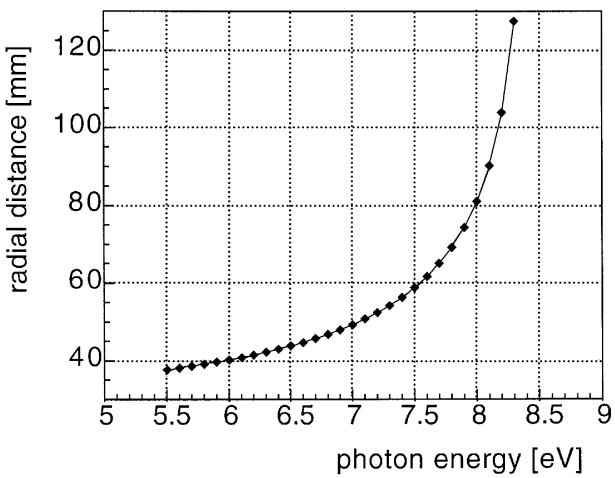

Fig. 3. Cherenkov photons distance from the ring centre as a function of photon energy, calculated for $2 \mathrm{GeV} / c$ protons, $2 \mathrm{~mm} \mathrm{NaF}$ radiator and $28 \mathrm{~mm}$ proximity gap.

be calculated as

$N_{\text {phot }}(E)=370 T_{\mathrm{r}} \int_{E}^{E+\mathrm{d} E} \sin ^{2} \theta_{\mathrm{c}}(E) \prod_{i} T_{i}(E) \mathrm{d} E$

where $T_{\mathrm{r}}$ is the radiator thickness, $\theta_{\mathrm{c}}$ is the Cherenkov angle and $T_{i}$ is the transmission of the $i$ th traversed medium. In the setup used, the upper limit of the spectral acceptance is set by the collection electrode frame (Fig. 1) which absorbs Cherenkov photons of energy above a value depending on the particle momentum $(8 \mathrm{eV}$ in the case of $2 \mathrm{GeV} / c$ protons); the lower limit is set by the CsI photoelectric threshold. Because of the large $\mathrm{NaF}$ chromaticity the Cherenkov fiducial zone can be subdivided into circular areas such that when a photon is counted in the $n$th zone its energy falls in a bin of width $\Delta E$ centred at $E_{n}$. Fig. 3 shows the dependence of the radial distance of the photon impact from the particle impact point as a function of the emitted photon energy. The values were calculated for photons produced by $2 \mathrm{GeV} / c$ proto$\mathrm{ns}$, a proximity gap of $28 \mathrm{~mm}$ and an emission point in the middle of a $2 \mathrm{~mm} \mathrm{NaF}$ radiator.

Assuming that all the quantities depending on energy are constant in each interval of width $\Delta E=$ $0.1 \mathrm{eV}$, the QE can be calculated with the approximated relation

$Q E\left(E_{i}\right)=\frac{N_{\mathrm{phel}}\left(E_{i}\right)}{370 T_{\mathrm{r}} \sin ^{2} \theta_{\mathrm{c}}\left(E_{i}\right) \prod_{j} T_{j}\left(E_{i}\right) \Delta E \varepsilon_{\mathrm{det}}}$

where $N_{\text {phel }}\left(E_{i}\right)$ is the number of photoelectrons counted in the $i$ th interval and $\varepsilon_{\text {det }}$ is the single-

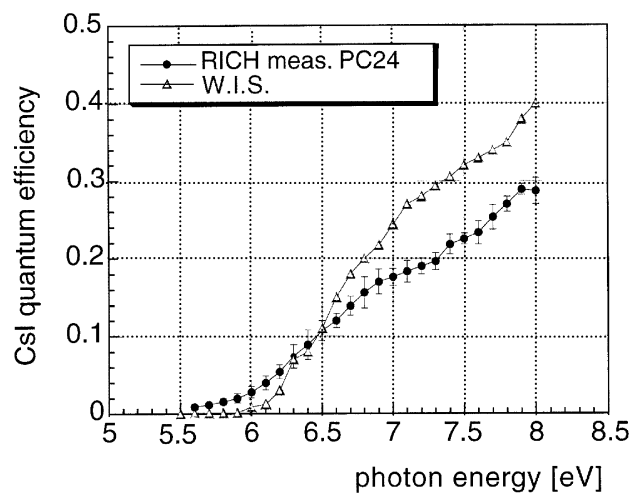

Fig. 4. PC24 QE, deduced from the analysis of Cherenkov rings generated in a $\mathrm{NaF}$ radiator, and the CsI QE obtained at the W.I.S. from photocurrent measurement on a small sample evaporated on stainless steel substrate.

electron detection efficiency. The accuracy of this method is limited by the proximity focusing error, resulting in the contamination, in a given energy bin, of photons belonging to adjacent energy bins. To minimize this effect a radiator as thin as possible has to be used, allowing narrower energy bins to be defined. We present the results obtained using proto-1 equipped with $\mathrm{PC} 24$ and a $\mathrm{NaF}$ radiator $2 \mathrm{~mm}$ thick. Several runs have been taken in different combinations of proton beam momenta ( 2 and $3 \mathrm{GeV} / \mathrm{c})$ and proximity gaps $(24.5,28,33,43$ and $48 \mathrm{~mm}$ ). The average QE curve of these measurements is reported in Fig. 4 and, for comparison, the CsI QE of a small sample, evaporated on stainless steel substrate, measured at the Weizmann Institute of Science (W.I.S.) by Breskin et al. [4,5].

At energies below the CsI photoelectric threshold $(\sim 6 \mathrm{eV})$ a negligible $\mathrm{QE}$ is expected. The large values measured, shown in Fig. 4, could be attributed to the feedback photons originated by the minimum ionizing particle (MIP). Indeed these energy bins correspond to small radial distances from the MIP impact (Fig. 3). This interpretation is supported by the results obtained using the Monte Carlo simulation described in the next paragraph.

\subsection{Evaluation with Monte Carlo simulation of $\mathrm{C}_{6} \mathrm{~F}_{14}$ events}

In the original Monte Carlo simulation program RICHSIM all physical processes, from the 
Cherenkov emission to the signal induction on the photocathode pad plane, are taken into account. Several input parameters can be specified, in order to match the experimental conditions: type of Cherenkov radiator $\left(\mathrm{NaF}\right.$ or $\left.\mathrm{C}_{6} \mathrm{~F}_{14}\right)$, its thickness $T_{\mathrm{r}}$ and $\mathrm{UV}$-transmission in the $160-220 \mathrm{~nm}$ range, proximity gap width $T_{\mathrm{g}}, \mathrm{O}_{2}$ and $\mathrm{H}_{2} \mathrm{O}$ contamination (in ppm), CsI QE curve, detector rotation angles, mean pedestal and sigma for electronic noise, chamber gain, photon feedback rate. Only the most peculiar sections of the simulation will be described here.

\subsubsection{Cherenkov photon generation: the $C_{6} F_{14}$ refractive index}

The number of photons generated along the particle trajectory in the radiator is drawn from a Poisson distribution with mean $N_{\text {av }}$ given by the integral of the Frank-Tamm relation over the energy range of detector response. The emission point in the radiator is uniformly distributed while the Cherenkov photon energy is sampled from a density function

$f(E)=1-\frac{1}{n^{2}(E) \beta^{2}}$.

Then the Cherenkov angle is calculated from the radiator refractive index. For $\mathrm{C}_{6} \mathrm{~F}_{14}$, before the recent measurements done by Fokitis et al. from DELPHI [6], only Séguinot's data [7] were available in the far UV. The linear extrapolation, $n(E)=$ $a+b \cdot E$, of Séguinot's values to the range of interest (with $a=1.2177, b=0.00928 \mathrm{eV}^{-1}$ ) results in a bad fit of experimental data; on the contrary, they are well reproduced using in RICHSIM $a=1.177$, $b=0.0172 \mathrm{eV}^{-1}$, a parametrization in rather good agreement with DELPHI measurements (Fig. 5).

It should be noticed that the $\mathrm{C}_{6} \mathrm{~F}_{14}$ sample purity and temperature (coefficient $\mathrm{d} n / \mathrm{d} T=$ $-5 \times 10^{-4}{ }^{\circ} \mathrm{C}^{-1}$ given by the supplier) may play an important role in explaining discrepancies among different measurements.

\subsubsection{Photon propagation and photoconversion at the CSI photocathode}

Each Cherenkov photon is propagated through the detector, undergoing absorption and interactions at the media boundaries (Fig. 6).

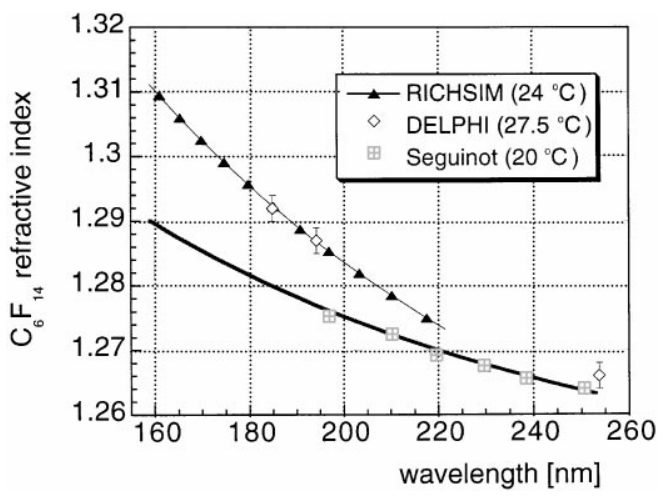

Fig. 5. The $\mathrm{C}_{6} \mathrm{~F}_{14}$ refractive index (DELPHI values by courtesy of E. Fokitis, S. Maltezos and P.G. Moyssides).

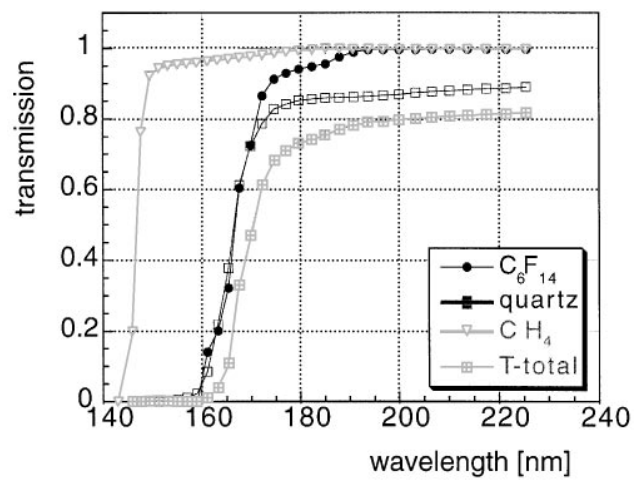

Fig. 6. Cherenkov photons transmission in detector media, calculated for $\beta=1$ particles, emission point at about half of the $10 \mathrm{~mm} \mathrm{C}_{6} \mathrm{~F}_{14}$ radiator, $5 \mathrm{~mm}$ quartz window (Heraeus) and $103 \mathrm{~mm}$ proximity gap. The total $T$ curve includes also the reflection losses at radiator/quartz interface, quartz/gas interface and electrodes.

At the point of photoconversion, Fresnel reflection losses on the CsI PC surface are taken into account to make the $\mathrm{PC}$ response varying with the photons incidence angle $\theta_{\mathrm{o}}$. The Fresnel reflection probablity is calculated using a complex refractive index for CsI [8]; then the following correction factor $S_{\mathrm{r}}$ for surface roughness is included [9]:

$S_{\mathrm{r}}=\exp ^{-4 \pi \cos \theta_{\mathrm{o}} \sigma_{\mathrm{r}} / \lambda}$

where $\lambda$ is the photon wavelength and $\sigma_{\mathrm{r}}$ is the surface roughness parameter (which is $\sim 20 \mathrm{~nm}$ in our case [2]). 


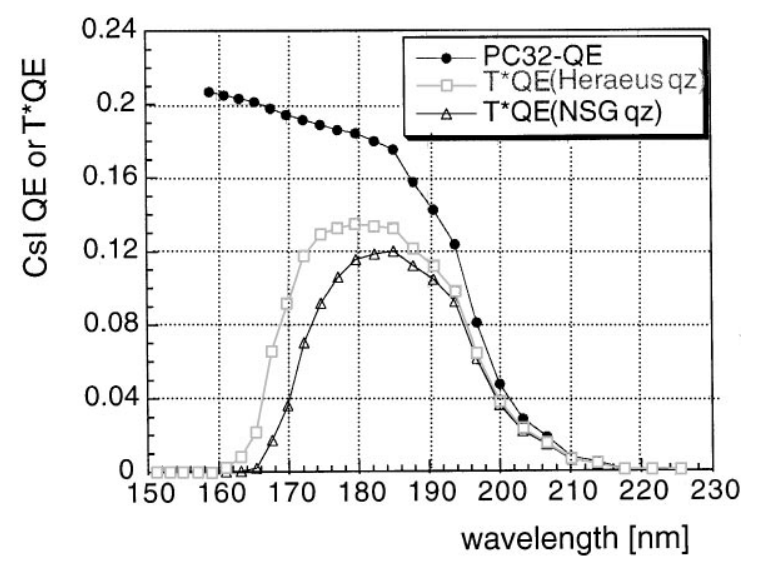

Fig. 7. PC32 QE evaluated from simulation and the convolution with the total transmission, defining the detected photon spectrum, obtained with two different quartz windows.

The PC32 QE used to reproduce test-beam data is shown in Fig. 7 together with the convolution with the total transmission curves obtained with two quartz windows of different quality, supplied by Heraeus and NSG.

\subsubsection{Generation of signal on pads and photon feedback}

The single-electron avalanche charge is drawn from an exponential distribution due to the $\mathrm{CH}_{4}$ and the moderate gain $\left(\leq 10^{5}\right)$ in use. Then a number $N_{\mathrm{pf}}$ of feedback photons is iteratively generated proportionally to the total avalanche charge $Q_{\mathrm{t}}: N_{\mathrm{pf}}=K_{\mathrm{pf}} Q_{\mathrm{t}} ; \quad K_{\mathrm{pf}}$ is the measured $\mathrm{CH}_{4}$ feedback rate [10] corrected for the so-called visible gain: the measured charge is smaller than $Q_{\mathrm{t}}$ due to both the signal sharing among electrode elements and the front-end electronics peaking time. The secondary photons are generated with an isotropic angular distribution from the avalanche position on the sense wire, sampling the three C emission lines: $156(30 \%), 166(57 \%)$ and $193 \mathrm{~nm}(13 \%)$ [11]. Incorporating the reflection at anode wires and at the CsI PC surface (including roughness) proved to be necessary for a correct simulation.

Finally, the electrostatic model used for the signal induction is based on the single-parameter Gatti formula $[12,13]$.

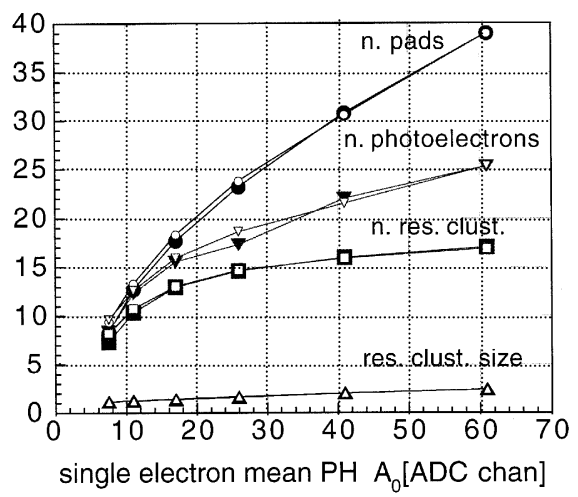

Fig. 8. Variation of main quantities of the Cherenkov ring analysis (number of pads/event, number of resolved clusters/event, resolved cluster size and number of photoelectrons/event) as a function of single electron mean $\mathrm{PH} A_{0}$ in proto-2, PC32, $350 \mathrm{GeV} / c \pi$ SPS test-beam (1 ADC channel corresponds to $0.17 \mathrm{fC}$ ). Full symbols: measurements; empty symbols: simulation.

\subsubsection{Electronic noise contribution and PH thresholding}

The electronic noise is generated on each pad with a Gaussian distribution having a standard deviation determined experimentally. Then a $\mathrm{PH}$ thresholding with pedestal subtraction produces the event that can be analysed by means of the same test-beam analysis software.

\subsubsection{Analysis results for chamber gain scan}

In Fig. 8 are reported the main multiplicities, measured and simulated, as a function of the single electron mean $\mathrm{PH} A_{0}$, namely the chamber gain, given in ADC channels (1 ADC channel corresponds to $0.17 \mathrm{fC}$, i.e. $\sim 1060 \mathrm{e}^{-}$). The measured number of photoelectrons is calculated as ratio between the total $\mathrm{PH}$ and $A_{0}$ while the simulation value is the true number of detected photoelectrons in RICHSIM events. The saturation in the resolved clusters multiplicity is due to the detection efficiency plateau while the continuous increase of the number of pad hits is related to the larger induced signal as well as to the increasing feedback photolectrons multiplicity. This interpretation is well supported by the plot in Fig. 9 showing the single contributions to the detected photoelectrons.

As a concluding remark, the simulation allowed to estimate the factor of merit $N_{0}=45 \mathrm{~cm}^{-1}$ with 


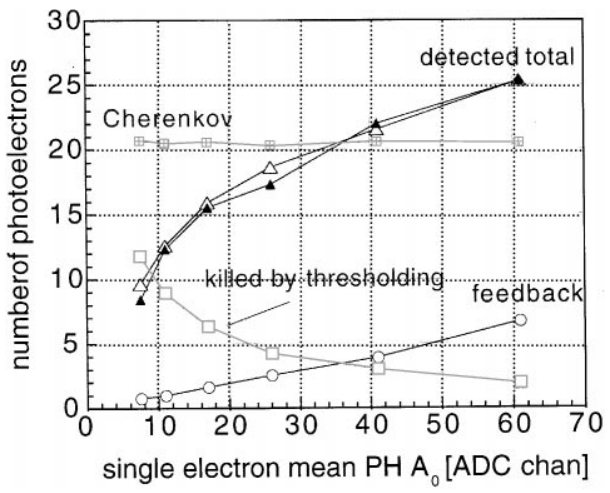

Fig. 9. The number of photoelectrons as a function of single electron mean $\mathrm{PH} A_{0}$ from beam test data is compared to the same quantity obtained from the simulation (1 ADC channel corresponds $0.17 \mathrm{fC}$ ). The single contributions to the total number of photoelectrons, estimated by simulation, are also reported: detected total $=$ Cherenkov + feedback - killed by thresholding.

proto-2/PC32 in typical operation conditions (chamber gain $A_{0}=41$ ADC channels, single-electron detection efficiency $\varepsilon_{\mathrm{det}}=90 \%$ ).

\section{Cherenkov angle resolution}

We have carried out an investigation of all the aspects influencing the spread of the reconstructed angle distribution, based on a combined analysis of beam test and Monte Carlo events as well as on the analytical estimation of single contributions: chromatic, geometric, localization and particle incidence angle [14].

The angle reconstruction procedure defines a relation $\theta_{\mathrm{c}}=\theta_{\mathrm{c}}\left(E, T_{\mathrm{r}}, R, \theta_{\mathrm{p}}\right)$ from which the angular resolution, referring to the adopted algorithm, can be analytically estimated as

$\sigma_{\theta_{\mathrm{c}}}=\left[\sum_{i=1}^{4}\left(\frac{\partial \theta_{\mathrm{c}}}{\partial v_{i}} \sigma_{v_{i}}\right)^{2}\right]^{1 / 2}$

where $\theta_{\mathrm{c}}$ is the Cherenkov angle, the variables $v_{i}$ : $E, T_{\mathrm{r}}, R, \theta_{\mathrm{p}}$ are the photon energy, the radiator thickness, the ring radius and the particle incidence angle, respectively, while $\sigma_{v_{i}}$ are the corresponding r.m.s. errors.

Two methods have been used for the Cherenkov angle reconstruction, in real and simulated events
[14]. Since the photon emission point in the radiator, $X_{\mathrm{ep}}$ and the photon energy are unknown, in both methods, the angle reconstruction is achieved by fixing them at the most probable values. In particular, $X_{\mathrm{ep}}$ is about $5.2 \mathrm{~mm}$ for perpendicular tracks in a $10 \mathrm{~mm}$ radiator; it differs from $T_{\mathrm{r}} / 2$ since photons generated in the first radiator half have a larger probability of being absorbed due to their longer path in the $\mathrm{C}_{6} \mathrm{~F}_{14}$. The average energy $E_{\mathrm{av}}$ is $6.85 \mathrm{eV}$ over the detector response (Fig. 7).

The first procedure, called $\beta$-method, is a direct transformation of the measured ring radius $R$ into a $\theta_{\mathrm{c}}$ value through a relation which can be established in case of perpendicular incidence and known $\beta$. Fixing the photon emission point and energy to the respective average values, the radiator and the quartz window contributions to $R, \Delta R_{\mathrm{rad}}$ and $\Delta R_{\mathrm{qz}}$, become constant terms and one can easily get $R_{\mathrm{o}}$ from $R=\Delta R_{\mathrm{rad}}+\Delta R_{\mathrm{qz}}+R_{\mathrm{o}}$. In turn $R_{\mathrm{o}}=T_{\mathrm{g}} \tan \theta_{\mathrm{o}}$ where $T_{\mathrm{g}}$ is the proximity gap thickness and $\theta_{\mathrm{o}}$ is the Cherenkov angle in the proximity gap. Then, using Snell's law and Cherenkov's relation to eliminate the dependence on the refractive index, $\theta_{\mathrm{c}}$ can be reconstructed as

$\theta_{\mathrm{c}}=a \cos \left(\frac{1}{\sqrt{\beta^{2} /\left(1+\left(T_{\mathrm{g}}^{2} / R_{\mathrm{o}}^{2}\right)\right)+1}}\right)$.

The second algorithm, the loop-method, is based on the search of the best angle through an iteration procedure described in Ref. [15].

Fig. 10 shows the reconstructed Cherenkov angle distributions relative to the two methods. A ring resolution of $1.9 \mathrm{mrad}$ has been obtained with the $\beta$-method, which, in principle, could be applied also to events where the particle track is not perpendicular (a simple detector rotation is needed), and when $\beta$ is not known (depending on the application, one has usually three mass hypothesis).

\subsection{Contributions to the Cherenkov angle resolution}

For the analytical treatment, after having obtained the partial derivatives $\left(\partial \theta_{\mathrm{c}} / \partial v_{i}\right)$ following Ypsilantis' formulation [16], the evaluation of the r.m.s. errors $\sigma_{v_{i}}$, strictly related to the detector response, is needed. 

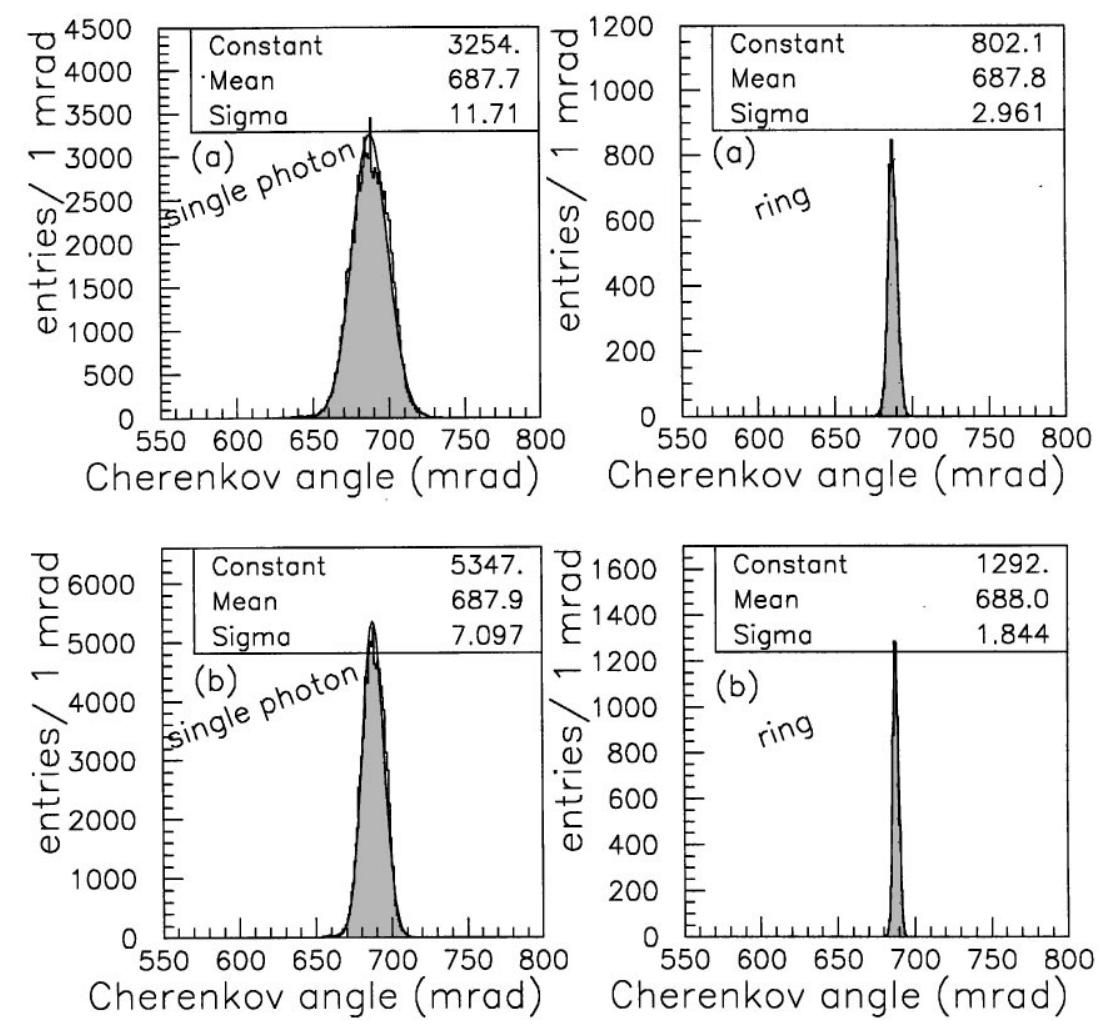

Fig. 10. Single-photon and ring-averaged, Cherenkov angle distributions, from (a) the loop and (b) the $\beta$-methods; SPS test-beam, $350 \mathrm{GeV} / c \pi$, PC32, single electron mean PH $A_{0}=41$ ADC channels (1 ADC channel corresponds to $0.17 \mathrm{fC}$ ).

The chromatic r.m.s. error is $\sigma_{E}=(\mathrm{d} n / \mathrm{d} E) \sigma_{E}^{\mathrm{det}}$, with $\mathrm{d} n / \mathrm{d} E=0.0172 \mathrm{eV}^{-1}$ and $\sigma_{E}^{\mathrm{det}}=1.75 / \sqrt{24} \mathrm{eV}$ (according to the detected photon spectrum reported in Fig. 7 for a Heraeus quartz window). For the geometric r.m.s. error $\sigma_{T_{\mathrm{r}}}$ the calculation is straightforward: $\sigma_{T_{\mathrm{r}}}=T_{\mathrm{r}} /\left(\sqrt{12} \cos \theta_{\mathrm{p}}\right)$. Finally, the total localization r.m.s. error $\sigma_{R}$ includes the uncertainty of both particle and photon $x, y$ coordinates: $\sigma_{R}=3 \mathrm{~mm}$. Such a large value, evaluated through RICHSIM, results from the poor localization accuracy achievable in single pad clusters ( $\sim$ pad size/ $\sqrt{12}$ ) as well as from the photon feedback contribution.

The results of the analytical estimation of each contribution in the two methods are reported in Table 1; for comparison, the same errors have been evaluated with RICHSIM, resulting in good agreement with the calculations.

\subsection{Optimization of the angular resolution in beam tests}

Different tests have been carried out to examine the angular resolution dependence on the Cherenkov ring radius, the chamber gain, the radiator thickness and the particle incidence angle $\theta_{\mathrm{p}}$.

The single errors, $\left(\partial \theta_{\mathrm{c}} / \partial v_{i}\right) \sigma_{v_{i}}$, and the singlephoton angular resolution, $\sigma_{\theta_{\mathrm{c}}}$ are reported in Table 2, from analytical calculation, simulation and measurements, for the $\beta$-method, at several ring radii. In Table 3 the same quantities, evaluated with the loop method, are shown. The $\sigma_{\theta_{\mathrm{c}}}$ simulation values come from the reproduction of test-beam runs, thus including effects related to the detector operating conditions specific to each run, while the calculated values refer to a constant configuration. Besides the good agreement among the different data sets one can notice that the deterioration at 
Table 1

Single-photon Cherenkov angle errors from analytical treatment and simulation $\left(\beta=1, T_{\mathrm{r}}=10 \mathrm{~mm}, T_{\mathrm{g}}=103 \mathrm{~mm}\right)$

\begin{tabular}{llllll}
\hline Error & Calculation & & & Simulation \\
\cline { 2 - 3 } \cline { 5 - 6 } & $\begin{array}{l}\beta \text {-method } \\
(\mathrm{mrad})\end{array}$ & $\begin{array}{l}\text { Loop-method } \\
(\mathrm{mrad})\end{array}$ & & $\begin{array}{l}\beta \text {-method } \\
(\mathrm{mrad})\end{array}$ & $\begin{array}{l}\text { Loop-method } \\
(\mathrm{mrad})\end{array}$ \\
\hline Chromatic & 5.7 & 9.4 & 5.9 & 9.7 \\
Geometric & 2.5 & 4.8 & 3.6 & 4.2 \\
Localization & 3.1 & 4.1 & 3.1 & 5 \\
\hline
\end{tabular}

Table 2

Single-photon extracted Cherenkov angle errors from analytical treatment, simulation and measurements, in the $\beta$-method, at several ring radii

\begin{tabular}{lllllll}
\hline $\begin{array}{l}\text { Ring radius } \\
(\mathrm{mm})\end{array}$ & \multicolumn{2}{l}{ Calculation } & & \multicolumn{2}{c}{ Simulation } & Measurement \\
\cline { 2 - 5 } & $\begin{array}{l}\text { Chromatic } \\
(\mathrm{mrad})\end{array}$ & $\begin{array}{l}\text { Geometric } \\
(\mathrm{mrad})\end{array}$ & $\begin{array}{l}\text { Localization } \\
(\mathrm{mrad})\end{array}$ & $\begin{array}{l}\sigma_{\theta_{c}} \\
(\mathrm{mrad})\end{array}$ & $\begin{array}{l}\sigma_{\theta_{c}} \\
(\mathrm{mrad})\end{array}$ & $\begin{array}{l}\sigma_{\theta_{c}} \\
(\mathrm{mrad})\end{array}$ \\
\hline 85 & 5.76 & 4.83 & 5.95 & 9.7 & 10.8 & 11.2 \\
104 & 5.73 & 3.78 & 4.66 & 8.4 & 8.2 & 8.6 \\
122 & 5.71 & 3.26 & 4.01 & 7.8 & 7.3 & 7.5 \\
133 & 5.7 & 2.9 & 3.6 & 7.43 & 7.4 & 7.6 \\
155 & 5.69 & 2.53 & 3.12 & 7.1 & 6.8 & 6.9 \\
\hline
\end{tabular}

Table 3

Single-photon extracted Cherenkov angle errors from analytical treatment, simulation and measurement, in the loop method, at several ring radii

\begin{tabular}{lllllll}
\hline $\begin{array}{l}\text { Ring radius } \\
(\mathrm{mm})\end{array}$ & \multicolumn{2}{l}{ Calculation } & & \multicolumn{2}{l}{ Simulation } & Measurement \\
\cline { 2 - 5 } & $\begin{array}{l}\text { Chromatic } \\
(\mathrm{mrad})\end{array}$ & $\begin{array}{l}\text { Geometric } \\
(\mathrm{mrad})\end{array}$ & $\begin{array}{l}\text { Localization } \\
(\mathrm{mrad})\end{array}$ & $\begin{array}{l}\sigma_{\theta_{c}} \\
(\mathrm{mrad})\end{array}$ & $\begin{array}{l}\sigma_{\theta_{c}} \\
(\mathrm{mrad})\end{array}$ & $\begin{array}{l}\sigma_{\theta_{c}} \\
(\mathrm{mrad})\end{array}$ \\
\hline 85 & 9.4 & 7.9 & 9.1 & 15.9 & 15.6 & 16.2 \\
104 & 9.4 & 6.2 & 7.1 & 13.8 & 13.3 & 13.8 \\
122 & 9.4 & 5.4 & 6.1 & 12.8 & 12.2 & 12.4 \\
133 & 9.4 & 4.8 & 5.4 & 12.2 & 12 & 12.4 \\
155 & 9.4 & 4.2 & 4.8 & 11.6 & 11.1 & 11.2 \\
\hline
\end{tabular}

$R<110 \mathrm{~mm}$ is mainly due to the dominance of the chromatic error. Such contribution could be reduced by the use of a quartz window having a larger wavelength cut-off. Fig. 7 presents the detected photons spectrum, obtained with Heraeus and NSG quartz windows. In the latter case, a $\sigma_{E}^{\text {det }}=1.4 / \sqrt{24} \mathrm{eV}$ can be estimated, decreasing the chromatic error by $20 \%$ with respect to that evaluated with the first quartz window. The drop in the number of photons can be compensated by a thicker Cherenkov radiator, with a small degradation of the resolution caused by the larger geometric error. Indeed the resolution evaluated by RICHSIM using the worse quartz transmission and $12.5 \mathrm{~mm}$ radiator is equal to the best values relative to the higher quality quartz and $10 \mathrm{~mm}$ 


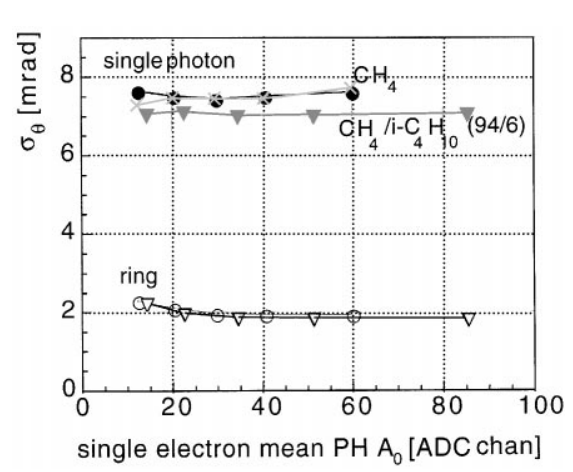

Fig. 11. Angular resolution as a function of the single-electron $\mathrm{PH} A_{0}$ at $R=122 \mathrm{~mm}$, in two gas mixtures; circles and triangles: measurements; crosses: simulation; SPS test-beam, $350 \mathrm{GeV} / c \pi$, PC32 (1 ADC channel corresponds to $0.17 \mathrm{fC}$ ).

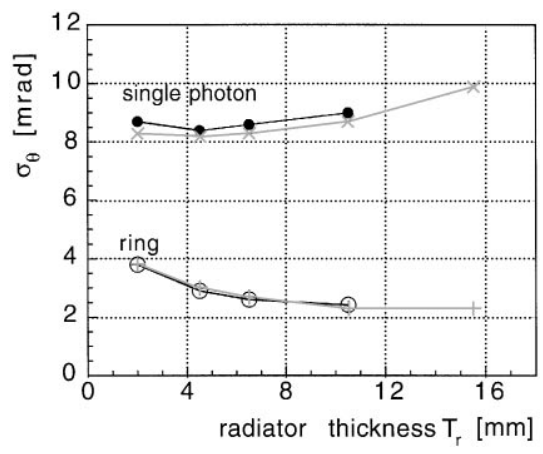

Fig. 12. Angular resolution as a function of radiator thickness at $R=104 \mathrm{~mm}$; circles: measurements; crosses: simulation; PS test-beam, $3 \mathrm{GeV} / c \pi$, PC24.

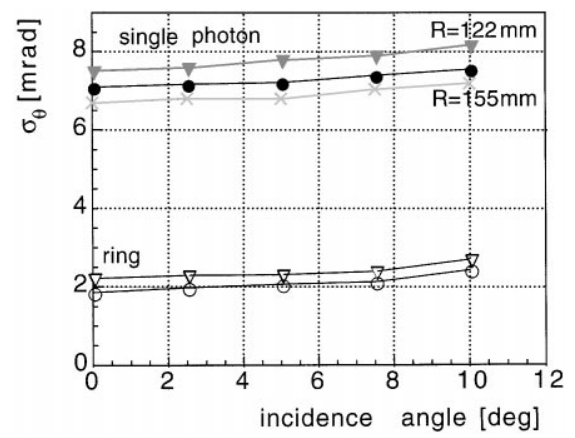

Fig. 13. Angular resolution as a function of the particle incidence angle at a single-electron mean $\mathrm{PH} A_{0}=41$ ADC channels (1 ADC channel corresponds to $0.17 \mathrm{fC})$; circles $(R=155 \mathrm{~mm})$ and triangles $(R=122 \mathrm{~mm})$ : measurements; crosses: $(R=155 \mathrm{~mm})$ simulation; SPS test-beam, $350 \mathrm{GeV} / c \pi$. The values for the ring radius $R=122 \mathrm{~mm}$ are relative to PC30 and a proximity gap $T_{\mathrm{g}}=80 \mathrm{~mm}$, while those for $R=155 \mathrm{~mm}$ are relative to PC32 and a proximity gap $T_{\mathrm{g}}=103 \mathrm{~mm}$.
$\mathrm{C}_{6} \mathrm{~F}_{14}$ (single photon: 7 mrad; ring: $1.9 \mathrm{mrad}$ ). As a consequence low-cost windows may be used, provided that the performance of the CsI PC could be kept at the standard level.

In Fig. 11 are reported the $\beta$-method singlephoton angular resolution $\sigma_{\theta_{\mathrm{c}}}$ and ring angular resolution $\sigma_{\theta_{c}}^{\text {ring }}$, from measurements and simulation, as a function of the single-electron mean $\mathrm{PH}$ $A_{0}$, in two gas mixtures. The stability of $\sigma_{\theta_{\mathrm{c}}}$ with the increase of $A_{0}$, at the considered ring radius, could be the result of two opposite effects: the larger feedback contribution, deteriorating the resolution, and the smaller fraction of single pad clusters, improving the resolution. In the mixture with $i-\mathrm{C}_{4} \mathrm{H}_{10}$ the single-photon resolution is slightly better than in pure $\mathrm{CH}_{4}$ probably due to the lower UV-transmission of $i-\mathrm{C}_{4} \mathrm{H}_{10}$, that was not circulated through any filter, reducing the chromatic error and the feedback contribution; however, the ring-averaged resolution is similar to that in pure $\mathrm{CH}_{4}$ because of the smaller number of Cherenkov photons.

Fig. 12 shows the $\beta$-method single-photon and ring-angular resolutions, from measurements and simulation, as a function of the radiator thickness $T_{\mathrm{r}}$. The small variations of $\sigma_{\theta_{\mathrm{c}}}$ are related to the changes of ring radius and radiator transmission with $T_{\mathrm{r}}$, while $\sigma_{\theta_{\mathrm{c}}}^{\text {ring }}$ increases, as expected, at smaller $T_{\mathrm{r}}$ as a consequence of the reduction of the number of emitted Cherenkov photons.

Finally, in Fig. 13 are reported the $\beta$-method single-photon and ring-angular resolutions, from measurements and simulation, as a function of the particle incidence angle $\theta_{\mathrm{p}}$. No significant deterioration of the resolution is observed up to $10^{\circ}$.

\section{Conclusions}

The described experimental procedures allowed us to estimate a fundamental detector parameter, the QE curve of large-area CsI PCs. The observed discrepancy with the values measured at the W.I.S. may result from the differences in the size of the samples, the nature of the PC substrates and the measurement procedures.

Other quantities, like the $\mathrm{C}_{6} \mathrm{~F}_{14}$ refractive index, the feedback photon rate and the different contributions to the Cherenkov angle resolution have 
been evaluated by means of the Monte Carlo program RICHSIM, demonstrating full understanding of the detector behaviour.

\section{Acknowledgements}

We are grateful to A. Braem, B. Goret, J. Raynaud and J.C. Santiard for their competent contribution to the development of the ALICE RICH detector. We also warmly acknowledge E. Fokitis, S. Maltezos and P.G. Moyssides for having put at our disposal their $\mathrm{C}_{6} \mathrm{~F}_{14}$ refractive index measurements. The author wishes to thank T. Ypsilantis for his availability in several useful discussions.

\section{References}

[1] ALICE Collaboration, Technical Proposal, CERN/LHCC 95-71.

[2] ALICE Collaboration, Technical Design Report of The High Momentum Particle Identification Detector, CERN/LHCC 98-19.
[3] M. Hempstead et al., Nucl. Instr. and Meth. A 306 (1991) 207.

[4] A. Breskin et al., Nucl. Instr. and Meth. A 367 (1995) 326.

[5] A. Breskin, Nucl. Instr. and Meth. A 371 (1996) 116.

[6] E. Fokitis, S. Maltezos, P.G. Moyssides, Measurements of the refractive index of DELPHI RICH radiators in liquid phase down to $185 \mathrm{~nm}$, in preparation.

[7] J. Séguinot, Les compteurs Cherenkov: applications et limites pour l'identification des particules. Developpements et perspectives. Ecole J. Curie de Physique Nucleaire, Mabuisson, France, September 1988.

[8] K.I. Said et al., J. Phys. C 10 (1977) 479.

[9] B.J. Stagg, T.T. Charalampopoulos, Appl. Opt. 30 (1991) 4113.

[10] R. Arnold et al., Nucl. Inst. and Meth. A 314 (1992) 465.

[11] H.D. Morgan et al., J. Chem. Phys. 60 (1974) 4734.

[12] E. Mathieson et al., Nucl. Instr. and Meth. A 227 (1984) 277.

[13] E. Mathieson et al., Nucl. Instr. and Meth. A 270 (1988) 602.

[14] A. Di Mauro, Internal Note ALICE 98-34.

[15] R. Arnold et al., Nucl. Instr. and Meth. A 270 (1988) 289.

[16] T. Ypsilantis, J. Séguinot, Nucl. Instr. and Meth. A 343 (1994) 30. 